\title{
TWO-STEP MECHANISM OF MACROMOLECULAR NUCLEATION AND CRYSTALLIZATION: FIELD THEORY AND SIMULATIONS
}

Pavel E. L'vov ${ }^{1}$ and Alexander R. Umantsev²

${ }^{1}$ Ulyanovsk State University, Ulyanovsk, Russia

${ }^{2}$ Fayetteville State University, Fayetteville, NC, USA

SI. Properties of Equilibrium Interface with Two Transition Parameters Equilibrium trajectory of an interface $\left\{X^{I}(x), \eta^{I}(x),\right\}$ between the old phase at $\left\{X^{\alpha}, \eta^{\alpha}\right\}$ and the new phase at $\left\{X^{\beta}, \eta^{\beta}\right\}$ is uniquely determined by Eqs. $(14,15)$ of the main text. In Figure $S 1$ are plotted interfacial trajectories in the phase space and the free energy densities along the trajectories. Notice that even equilibrium interfaces exhibit the two-step features manifested in the position of the energy density maximum on the trajectory. Moreover, depending on the interaction parameters, the first process to go is either compositional variation or ordering.

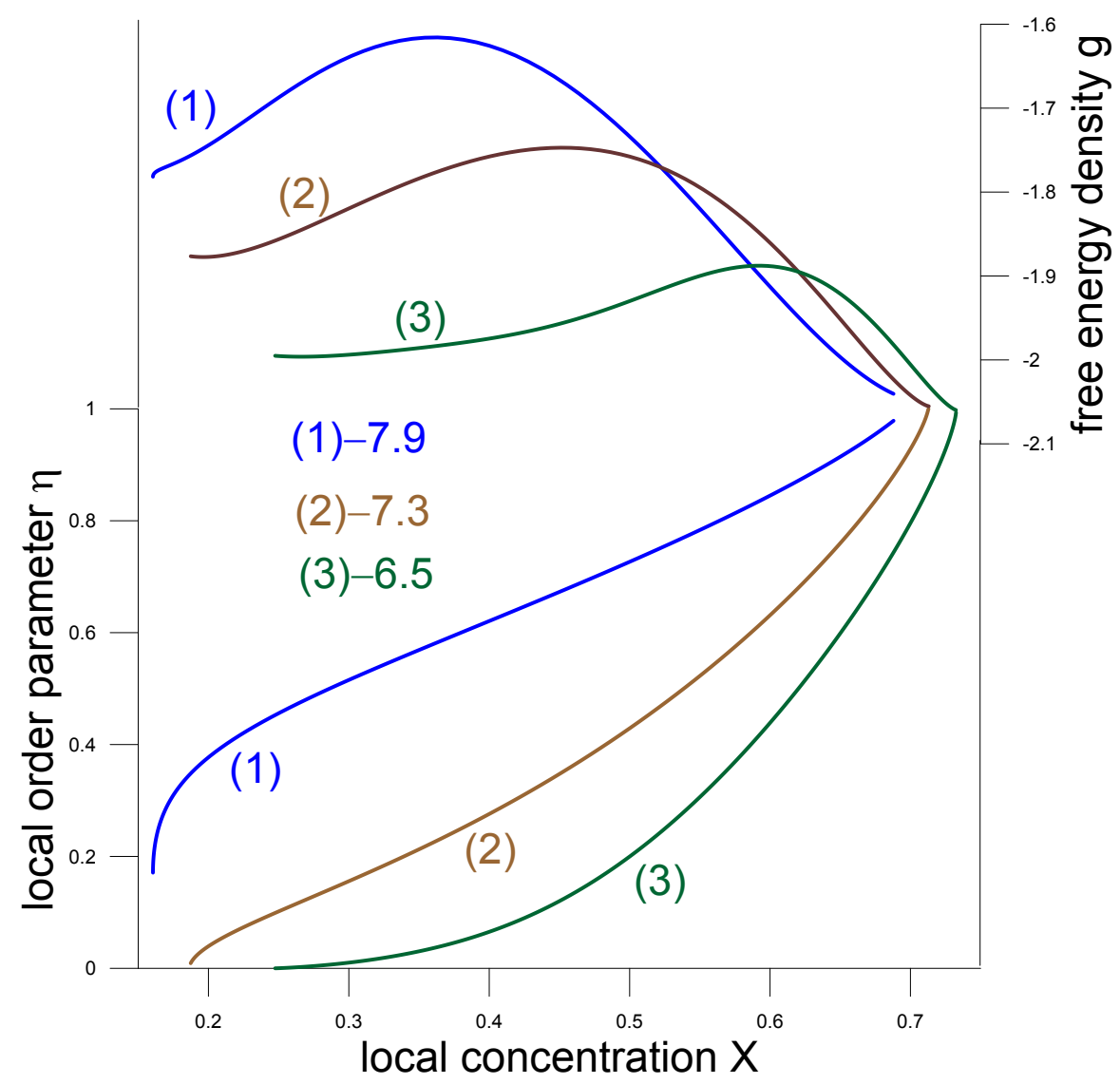

Figure S1. Phase space trajectories $(X, \eta)$ and free energy densities $\tilde{g}$ along the trajectories of the solid/liquid interfaces with various liquid interaction parameters $r^{l}$ shown on the plot. 
The interface has two fundamental properties - the interfacial energy

$\gamma=\int_{-\infty}^{+\infty}\left[\kappa_{X}\left(\frac{d X}{d x}\right)^{2}+\kappa_{\eta}\left(\frac{d \eta}{d x}\right)^{2}\right] d x$

and thickness $\mathrm{L}$. The latter may be defined by the maximum strength of the gradients of the transition parameters between the two homogeneous phases. For the transition between the $\alpha$-liquid $\left\{\eta^{l^{\alpha}}, X^{l^{\alpha}}\right\}$ and $\beta$-solid $\left\{\eta^{s^{\beta}}, X^{s^{\beta}}\right\}$ phases with two transition parameters, each one is characterized by its own thickness,

$L_{X} \equiv \frac{\left|X^{\beta}-X^{\alpha}\right|}{\max _{x}|d X / d x|}$

or

$L_{\eta} \equiv \frac{\left|\eta^{\beta}-\eta^{\alpha}\right|}{\max _{x}|d \eta / d x|}$

which do not need to be equal [S1]. It is instructive to compare the properties of the double interface (characterized by two transition parameters) with those of the single interface (characterized by one transition parameter, e.g. concentration). In Figure S2 are plotted the interfacial energies and thicknesses of the double and single interfaces with different liquid interaction parameters $\mathrm{r}^{l}$. Overall, the double interfaces are thinner and more energetic than their single counterparts because the former encompass variations of the concentration and ordering gradients while the latter- of the concentration gradients only, see Eqs.(S1,S2). Also notice that, at the approach of the critical point $r^{C} \approx 6.492$, the single (liquid/liquid) interface has familiar features of vanishing energy and diverging thickness while the double (liquid/solid) interface is not divergent. 

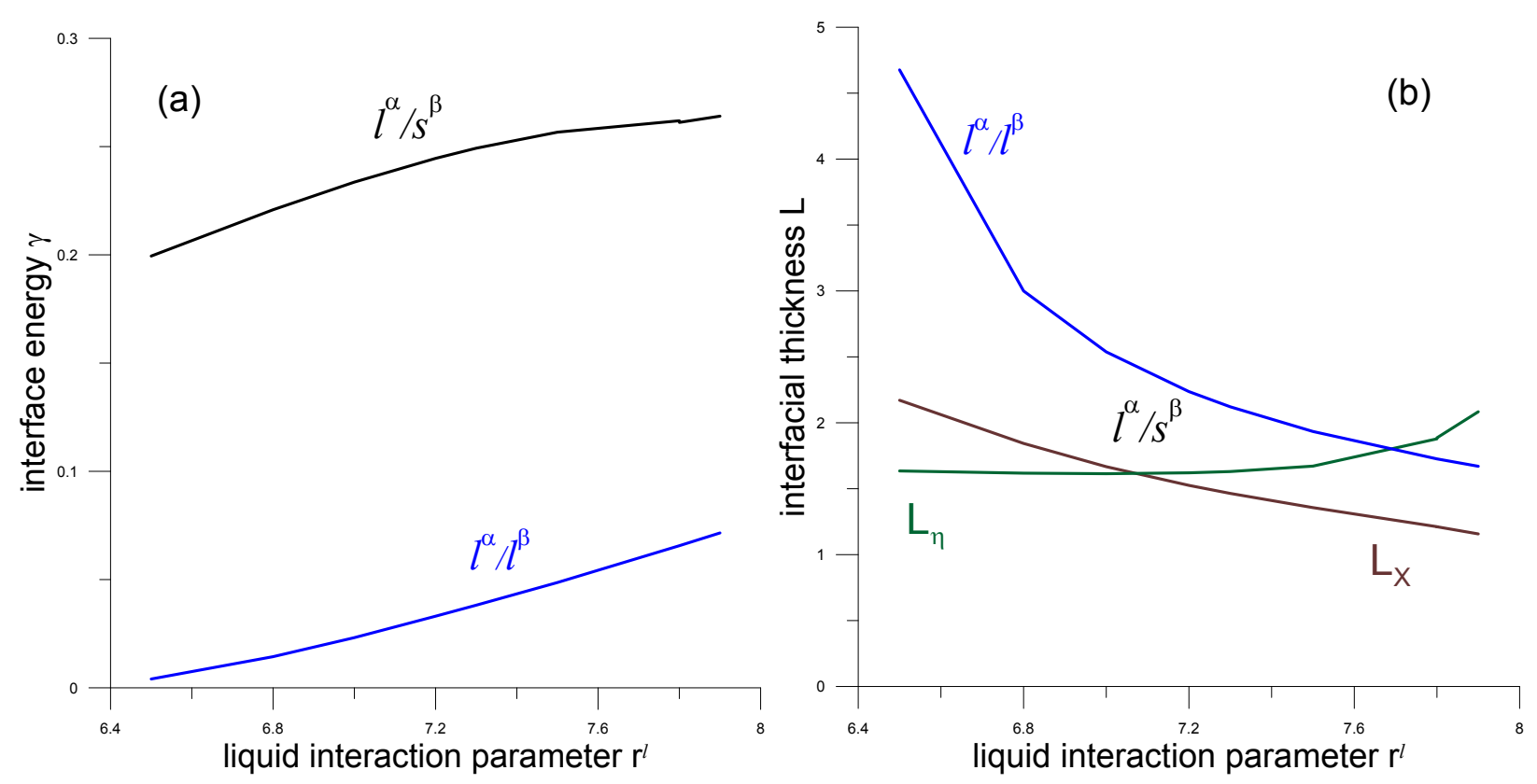

Figure S2. The interfacial energies $\gamma(\mathrm{a})$ and thicknesses $L$ (b) of the single (liquid/liquid) $\left(l^{\alpha} / l^{\beta}\right)$ and double (liquid/solid) $\left(l^{\alpha} / s^{\beta}\right)$ interfaces in solutions with different liquid interaction parameters $r^{l}$. The units are dimensionless. For the definitions and designations, see Eqs.(S1,S2) and Sec. $V$ of the main text.

SII. Numerical Method

To simulate crystallization of macromolecules, we solve the coupled dynamic equations $(19,20)$ of the main text in 3D with periodic boundary conditions using the semi-implicit Fourier spectral method [S2]. Specifically, we calculate the time evolution of the Fourier transforms of the concentration $\hat{X}_{\mathbf{k}}^{n}$ and order parameter $\hat{\eta}_{\mathbf{k}}^{n}$ by the following numeric scheme

$$
\begin{aligned}
& \hat{X}_{\mathbf{k}}^{n+1}=\frac{\hat{X}_{\mathbf{k}}^{n}-\Delta t \hat{g}_{X \mathbf{k}}^{n}|\mathbf{k}|^{2}}{1+\Delta t|\mathbf{k}|^{4}}+\zeta_{\mathbf{k}}^{\prime}, \\
& \hat{\eta}_{\mathbf{k}}^{n+1}=\frac{\hat{\eta}_{\mathbf{k}}^{n}-\Delta t S \hat{g}_{\eta \mathbf{k}}^{n}}{1+\Delta t D|\mathbf{k}|^{2}}+\xi_{\mathbf{k}}^{\prime} .
\end{aligned}
$$

where $\hat{g}_{X \mathbf{k}}^{n}$ and $\hat{g}_{\eta \mathbf{k}}^{n}$ are the Fourier transformations of the functions $\partial_{X} g$ and $\partial_{\eta} g$, respectively and $\mathbf{k}$ is the wave vector. The superscript $n$ determines the time moment $t=n \Delta t$ where $\Delta t$ is 
the time step. The stochastic variables $\zeta_{\mathbf{k}}^{\prime}$ and $\xi_{\mathbf{k}}^{\prime}$ are described by the following correlation functions [S3-S6]:

$\left\langle\zeta_{\mathbf{k}}^{\prime}, \zeta_{\mathbf{k}^{\prime}}^{\prime}\right\rangle=\frac{2 K_{X} \Delta t}{N}\left(3-\cos \left(k_{x} \Delta x\right)-\cos \left(k_{y} \Delta y\right)-\cos \left(k_{z} \Delta z\right)\right) \delta_{\mathbf{k},-\mathbf{k}^{\prime}}$

$\left\langle\xi_{\mathbf{k}}^{\prime}, \xi_{\mathbf{k}^{\prime}}^{\prime}\right\rangle=\frac{K_{\eta} \Delta t}{N} \delta_{\mathbf{k},-\mathbf{k}^{\prime}}$

Utilization of the white noise for the simulation of stable and metastable states in our systems did not yield formation of the nuclei. That is why we used the wave vector cut-off $k_{\text {max }}$ such that $\zeta_{\mathbf{k}}^{\prime}=\xi_{\mathbf{k}}^{\prime}=0$ for wave vectors with $|\mathbf{k}|>k_{\max }$. The wave vector cut-off related to the correlation length of fluctuation [S5, S7].

Computations by Eqs. $(\mathrm{S} 3, \mathrm{~S} 4)$ provide detailed dynamic information about the concentration and order parameter distributions over the entire volume of the system. Individual clusters that is, dense liquid droplets and crystalline particles were characterized by their volume-averaged concentrations $\left\langle X_{i}\right\rangle$, order parameter values $\left\langle\eta_{i}\right\rangle$, free energy densities $\left\langle\tilde{g}_{i}\right\rangle=\left(\int_{V_{i}} \tilde{g} d V\right) / V_{i}$, and equivalent radii $R_{i}=\left(3 N_{i} / 4 \pi\right)^{1 / 3}$ where $N_{i}$ is the number of grid points associated with the $i$ th cluster, which is proportional to the volume $V_{i}=N_{i} l^{3}$ (in dimensional variables). Simulations were performed in the volume of $V=256 \times 256 \times 512$ grid points with the dimensionless time step of $\Delta t=10^{-3}$ using the wave vector cut-off $k_{\max }=1.62$. The initial state of the system was characterized by the Gaussian distribution of the solute concentration $\left(E[X]=X_{0}, D[X]=10^{-4}\right)$ and order parameter $\left(E[\eta]=0, D[\eta]=10^{-4}\right)$. During the simulations, the average values of the concentration $\langle X\rangle$ remained equal to the initial overall value $X_{0}$ with very high accuracy.

Identification of an individual cluster of minority phase was performed by the method of nearest neighbor, which requires a threshold value for the grid points to be associated with the cluster. In the present work, we used $X_{t h}=0.35$ for the liquid droplets and $\eta_{t h}=0.25$ for the crystalline particles. The method is widely used in the analysis of phase distributions in atom probe tomography $[S 7,58]$. The method of nearest neighbors allowed us to identify subcritical, critical, and supercritical nuclei and eliminated the need to calculate the particle-size distribution function. However, characterizing individual clusters at a limited number of time instants did not 
allow us to observe the fast stages of the process, like passing of a nucleation barrier or initiation of crystallization. To offset this problem, we assumed ergodicity of the system and analyzed the properties of the whole ensemble of clusters at all moments of time [S5]. Such analysis gave us the dynamic trajectories of the clusters and allowed for visualization of the nucleation barrier, see Figs.7-9 of the main text. For the simulated parameters of the solution, the critical clusters were rather diffused. Due to high level of fluctuations below the threshold values of concentration and ordering, direct calculation of the work of formation of the critical clusters was not possible. Instead, we calculated energies of the truncated clusters above the threshold values and compared them with the theoretical estimates of the same quantities.

SIII. Heterogeneous Nucleation of Crystals

Dense liquid

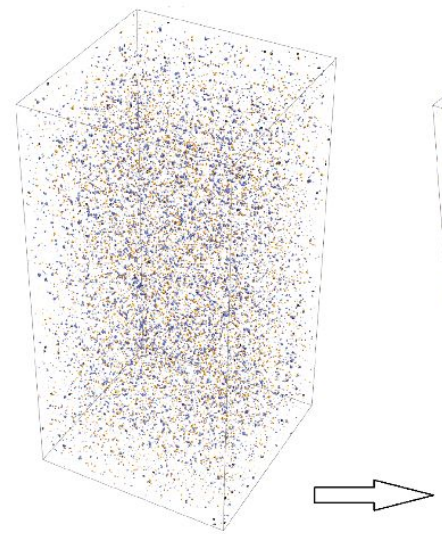

Nucleation

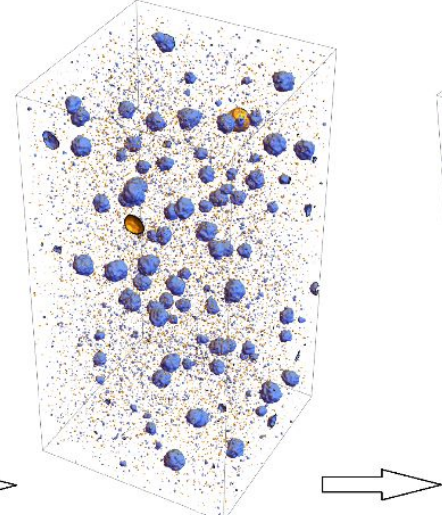

Crystallization
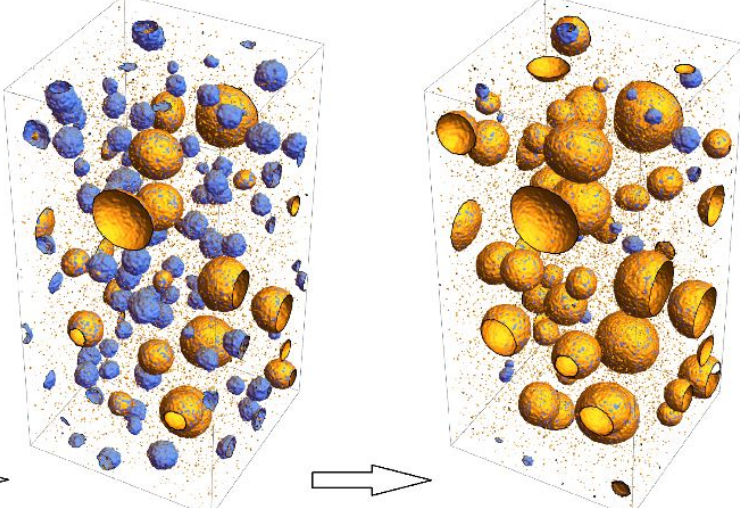

Maturation

Figure S3. Three steps of the transformation from the low-density liquid state to high-density crystallites in a solution of macromolecules. Blue particles-dense liquid droplets; yellow particles-crystallites. 
(a)

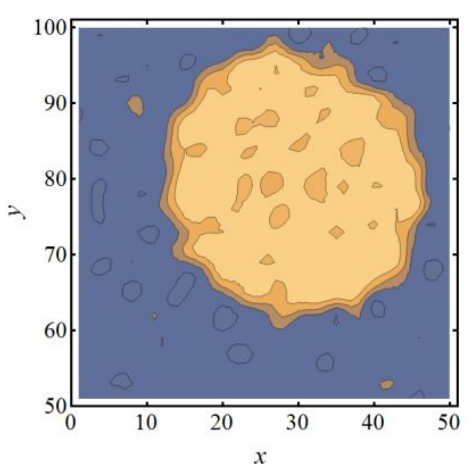

(d)

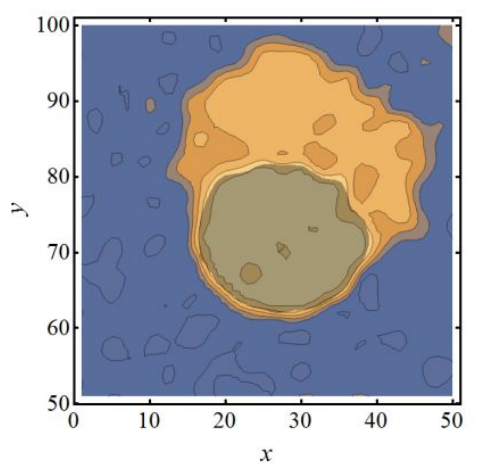

(b)

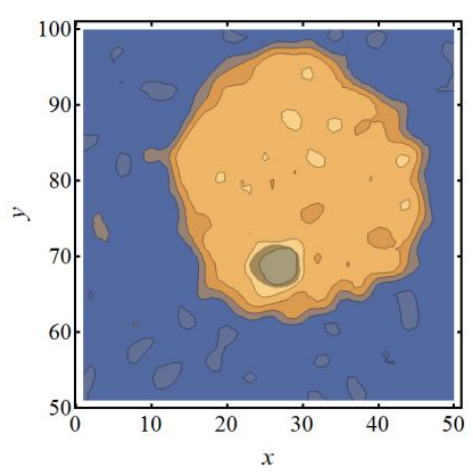

(e)

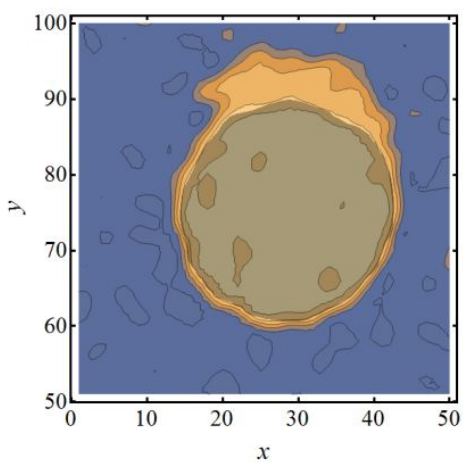

(c)

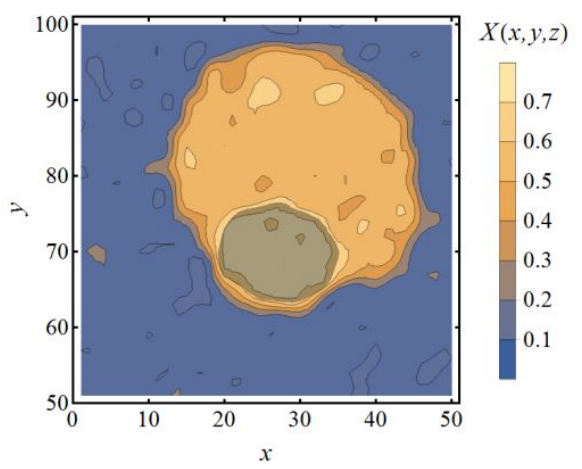

(f)

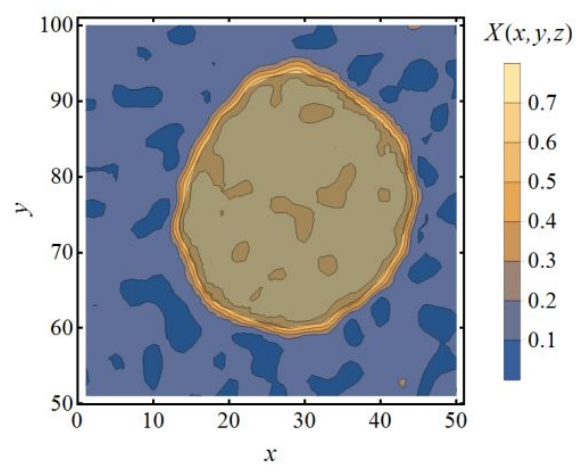

Figure S4. Overlapping snapshots of the concentration $X(x, y, z)$ (scale bar on the side) and ordering $\eta(x, y, z)$ (gray color, $\eta>0.5$ ) fields during crystallization of a single droplet of the system depicted in Fig. S3 at $z=411$ and (a) $t=1410$ (dense liquid droplet), (b) $t=1440$ (heterogeneous nucleation of a crystal), (c) $t=1460$, (d) $t=1480$, (e) $t=1510$, (f) $t=1530$ (crystalite). Cf. Figure 6 of the main text. 
(a)

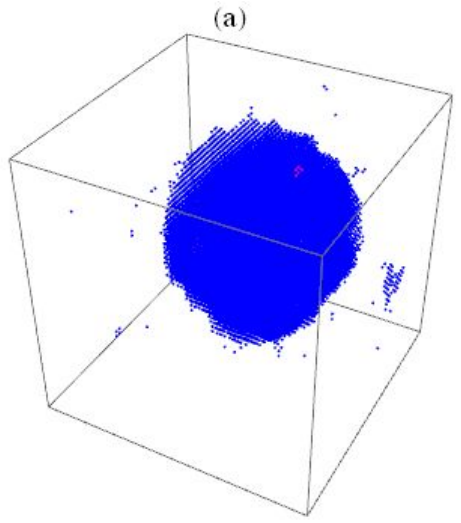

(d)

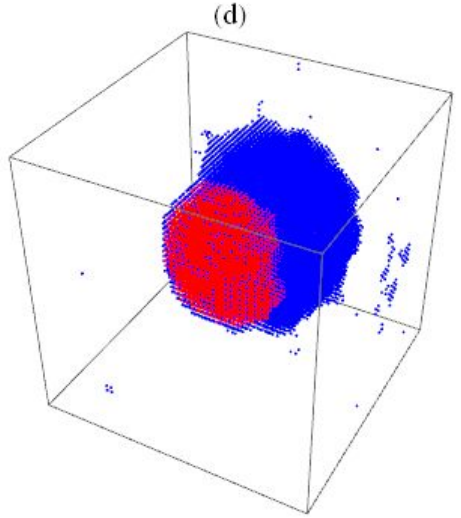

(b)

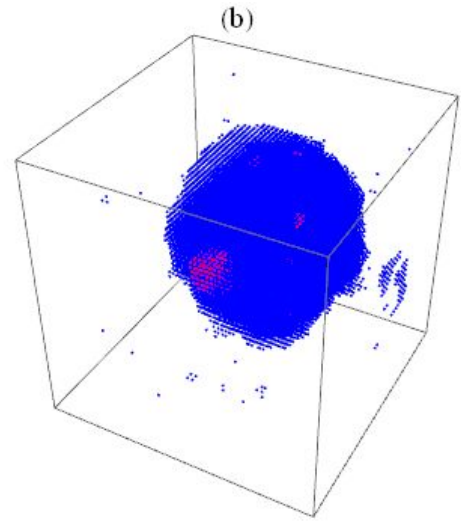

(e)

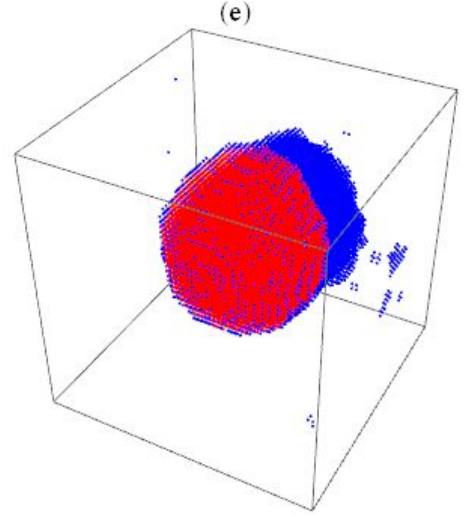

(c)
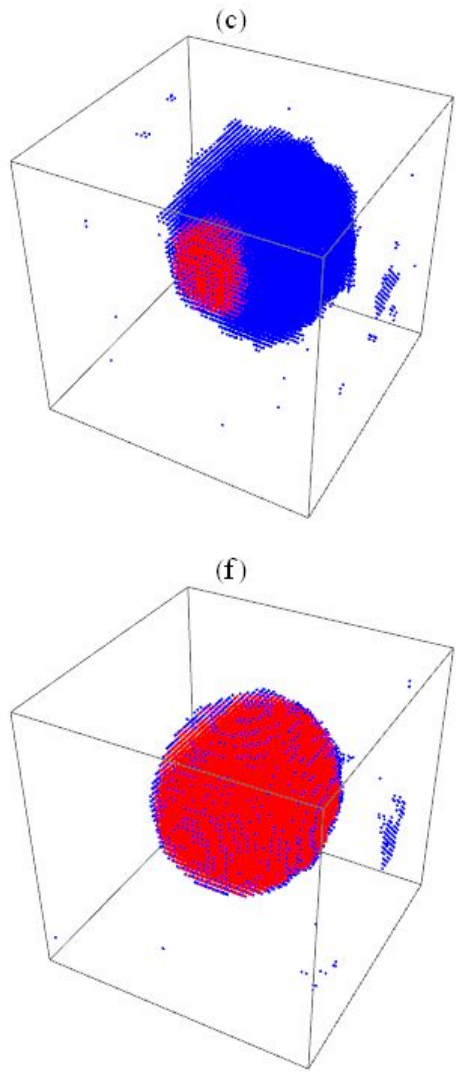

Figure S5. 3D reconstruction map of crystallization of the nucleus in Figure S4. Blue-liquid state domain of high density: $X \geq 0.35, \eta<0.25$; red-the domain of crystallized state with $X>0.35, \eta \geq 0.25$. The processes of heterogeneous crystal nucleation and particle contraction are clearly visible.

Movie S1. Dynamics of crystallization in a solution of macromolecules with the interaction strength $r^{l}=7.2$ and overall concentration $X_{0}=0.253$. Blue particles-dense liquid droplets; yellow particles-crystallites. The concentration field is shown by the blue level surface of $X(x, y, z)=0.4$, the order parameter filed-by the yellow level surface of $\eta(x, y, z)=0.35$.

Movie S2. Dynamics of crystallization in a solution of macromolecules with the interaction strength $r^{l}=7.3$ and overall concentration $X_{0}=0.253$. Blue particles-dense liquid droplets; yellow particles-crystallites. The concentration field is shown by the blue level surface of $X(x, y, z)=0.4$, the order parameter filed - by the yellow level surface of $\eta(x, y, z)=0.35$. 


\section{References}

S1. Umantsev, A. Identification of material parameters for modeling of phase transformations, Phys. Rev. B 2007, 75, 024202.

S2. Chen, L.-Q.; Shen, J. Application of Semi-Implicit Fourier-Spectral Method to Phase Field Equations, Computer Phys. Comm. 1998, 108, 147.

S3. Elder, K. R.; Rogers, T. M.; Desai, R. C. Early Stages of Spinodal Decomposition for the CahnHilliard-Cook Model of Phase Separation. Phys. Rev. B 1988, 38, 4725-4739.

S4. Garsia-Ojalvo, J.; Sancho, J. M. Noise in Spatially Extended Systems. Springer: New York, 1999.

S5. L'vov P. E.; Svetukhin, V. V. Simulation of the First Order Phase Transitions in Binary Alloys with Variable Mobility. Modelling Simul. Mater. Sci. Eng. 2017, 25, 075006.

S6. L'vov, P. E., Svetukhin, V. V. Stochastic simulation of nucleation in binary alloys. Modelling Simul. Mater. Sci. Eng. 2018, 26, 045001.

S7. L'vov, P. E., Svetukhin, V. V. Generalized non-classical nucleation model in binary alloys Modelling Simul. Mater. Sci. Eng. 2019, 27, 025002.

S8. Miller, M.; Forbes, R. Atom-Probe Tomography: The Local Electrode Atom Probe. Springer: New York, 2014. 